\title{
Paper
}

\section{Classification and Matching Method for Poultry Products using Inorganic Components}

\author{
Motoki GODA*†‡ Member, \\ AKIHIRO NOMURA ${ }^{\ddagger}$ Non-member, \\ SHUNSUKE IWANAMI ${ }^{\S}$ \\ Non-member \\ YOHICHIRO KOJIMA ${ }^{\dagger}$ Member
}

(Received September 21, 2021, revised January 6, 2022)

\begin{abstract}
It is essential to ensure the safety of food products against the backdrop of recent incidents related to food falsification. We have investigated the possibility of classification matching mainly for meat products, and in a previous study, we focused on classification matching by inorganic components for pork products. In this study, we investigated whether it is possible to classify and match the inorganic components of poultry products by learning them using a neural network. We demonstrated that the inorganic content of poultry products can be used to classify and match poultry products to their parts and that a neural network can be used to discriminate products with unknown parts.
\end{abstract}

Keywords: Poultry product, Classification, Inorganic components, Neural network, ROC curve

\section{Introduction}

To ensure food safety, many chemical analyzers have been used to evaluate food composition. There has been an urgent need to ensure safety in recent years due to various factors, such as an increase in imported foods, food production areas, food fraud, and detection of food poisoning incidents. Against this background, it has become important to develop the ability to quickly match the analytical results for many foods and classify them accurately.

Traditionally, the amounts of various chemical components in foods are quantified using chemical analyzers such as atomic absorption spectrometry, X-ray fluorescence diffractometry, gas chromatography/mass spectrometry, and HPLC.[1] [3] However, these methods' quantitative and accumulated data have generally been used only for nutritional aspects, not for classification and matching of food types and forms, which is becoming increasingly important currently. Therefore, this research group is investigating the possibility of optimizing and facilitating the classification and matching of foods using the chemical content of foods as quantified by quantitative chemical methods. Previous studies examined pork, beef, and products from other four-legged animals, and possibilities for classification and matching have been reported, mainly through multivariate analysis. However, in this context, there is a lack of studies on animal products other than those from four-legged animals, such as poultry.[4] [7]

\footnotetext{
* Corresponding: gouda@njpc.co.jp

$\dagger$ Hokkaido University of Science

7-Jo 15-4-1, Maeda, Teine, Sapporo, Hokkaido, Japan 006-8585

¥ North Japan Port Consultants Co., Ltd.

S2E2, 8-1, Sapporo, Hokkaido, Japan 060-0052

$\S$ National Institute of Technology, Tomakomai College

443 Aza-Nishikioka, Tomakomai, Hokkaido, Japan 059-1275
}

Therefore, this study investigated whether the inorganic components (sodium, potassium, calcium, magnesium, phosphorus, iron, zinc, and copper) in poultry products can classify and match the different parts.

\section{Materials and Method}

2.1 Poultry products as analysis data This study was conducted using the data of 38 poultry products published by the Ministry of Education, Culture, Sports, Science, and Technology. Using the amounts of inorganic components obtained by various instrumental analyses, these products were classified and collated into nine parts. [1]

2.2 Date preparation considering analysis error In addition to the original data for 38 poultry products, data for inorganic ingredients were prepared, accounting for analysis errors. Data for a total of $76(+10 \%$ : 38 products, $-10 \%$ : 38 products) products were added, with measurement errors of 8 inorganic components within $\pm 10 \%$. This protocol made it possible to consider the expected measurement errors when measuring the inorganic components in 38 different poultry products.

\subsection{Calculation of discrimination rate by neural net-} work Using the data on the amounts of the eight inorganic ingredients present, we verified whether poultry products could be classified and matched according to parts using the discrimination rate of a neural network.

A neural network is a mathematical model representing neurons in the human brain. The model consists of an input layer, an output layer, and a hidden layer. The information received in the input layer is evaluated in the output layer, and the appropriate information is outputted in each category (product type, part, and cooking method). A differentiable activation function is used in the hidden layer to 


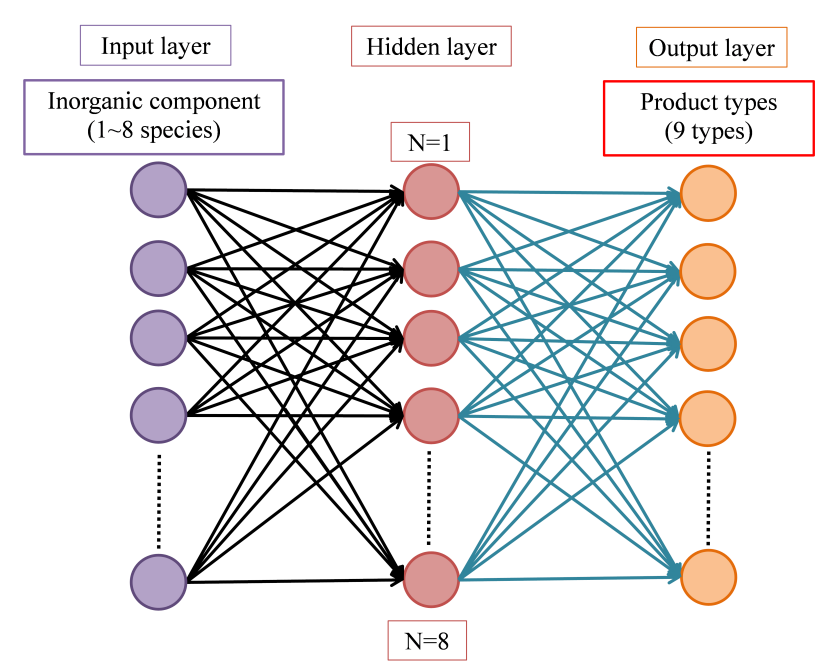

Figure 1: Image of neural network using inorganic components.

Table 1: Product parts of poultry products.

\begin{tabular}{l}
\hline Product parts \\
\hline Wing \\
Breast \\
Thigh \\
Ground meat \\
Offal and by-products \\
Meat substitute \\
Wing tip \\
Wing stick \\
\hline
\end{tabular}

define the output data at the output. [8] [9]

Calculations were performed using the statistical analysis software Weka (Waikato University), the Windows 10 operating system, a memory of $8 \mathrm{~GB}$, and an Intel Core i5-8500 processor.

The discrimination rate was determined using the following Eq. 1. It can be expressed by dividing the number of samples correctly predicted for each category as defined by the neural network by the total number of samples. In this report, we present the results of an experiment conducted with eight intermediate layers and 0-25,000 training cycles.

$$
\begin{aligned}
& \text { Determination rate }(\%) \\
& =\frac{\text { Positive discriminated samples }}{\text { All samples }} \times 100
\end{aligned}
$$

The calculations of the neural network were performed on each of the 38 types of data without considering the measurement error and on 114 types of data with consideration of the measurement error, and the change in the positive discrimination rate was confirmed.

\subsection{Validation of multiclass classification and ROC}

curve Classification and matching trends were confirmed based on the neural network results for the parts of the products using the amounts of eight inorganic chemical components. The measured and predicted values for each part were checked using a multiclass classification method, and the ratio of the predicted part to the known part and the predicted reproduction ratio were calculated. For example, if the correct part is "chicken wing," the ratio of the number of samples that were correctly identified as "chicken wing" by the neural network is calculated using Eq. 2. Recall is the predicted reproduction ratio of "part A", $T A$ is the number of samples that were correctly identified, $F X_{(X=n)}(A)$ is the number of samples that were incorrectly identified as "parts other than A", and $n$ is the number of other "parts".

The calculated predicted repeatability ratio Recall and false-positive rate $F T P$ for each part were plotted, and ROC curves for each part were generated.

$$
R_{A}=\frac{T A}{A+F A(A)+F C(A)+\cdots+F X_{(X=n)}(A)}
$$

\section{Results and Discussion}

Figure 2 shows the positive discrimination rate of the neural network using the inorganic component amount data of the poultry products. An evaluation including and excluding measurement errors was made and compared.

As shown in Fig. 2, the results without measurement error show the highest positive discrimination rate of $63.2 \%$ at 5,000 training cycles. Subsequently, the positive discrimination rate decreased with an increase in the number of training cycles, and it decreased to $55.3 \%$ at 25,000 cycles. This may be because the number of samples was small, and overlearning occurred once the number of training cycles exceeded 5,000.

The measurement errors exceeded a $90.0 \%$ positive discrimination rate after 15,000 training cycles, indicating the possibility of highly accurate classification and matching of poultry product parts. The highest positive discrimination rate was $92.1 \%$ at 20,000 training cycles, after which the rate began to decrease.

The number of samples with no measurement error was 38 , which is insufficient for examining the possibility of classification and matching by the neural network and is

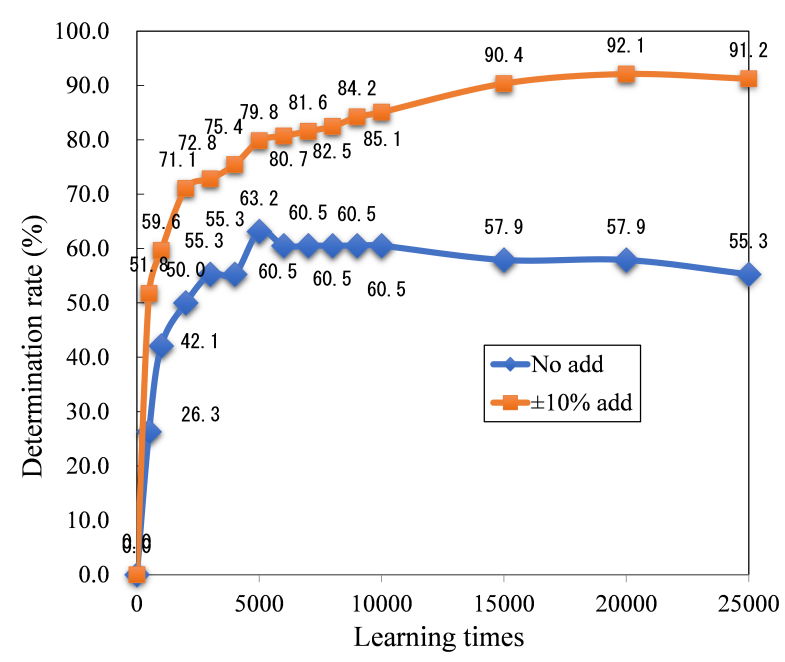

Figure 2: Results of neural network analysis using inorganic components in poultry products. 


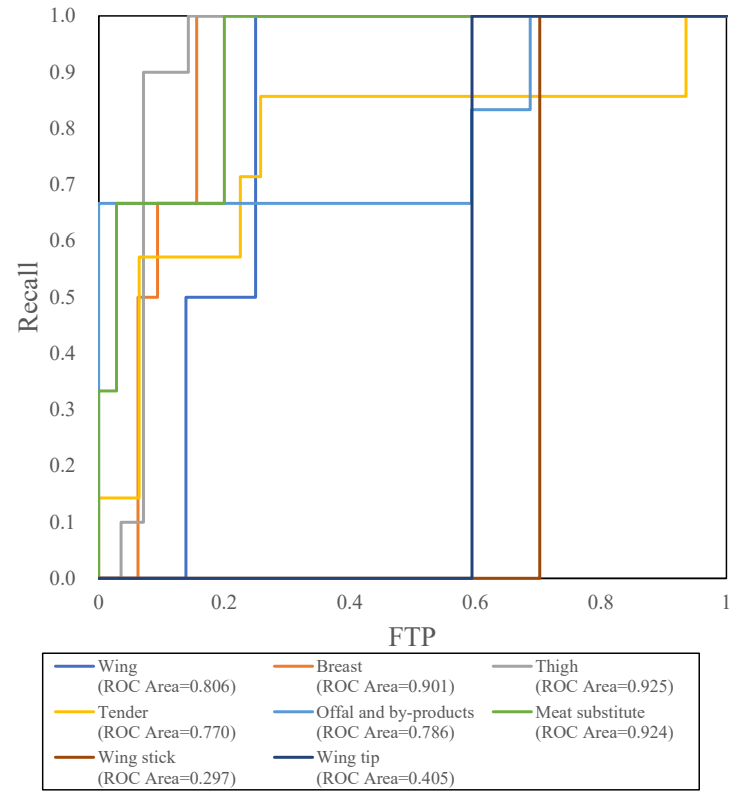

(a) Without error

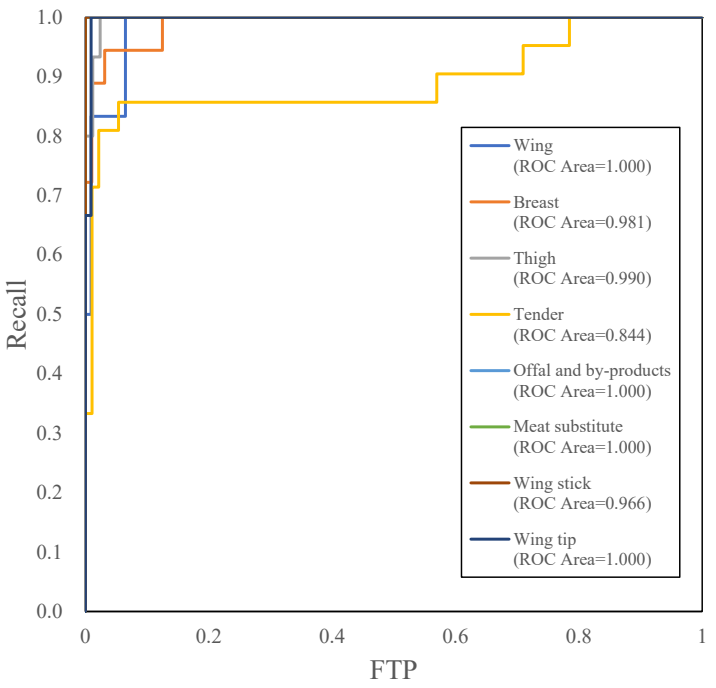

(b) With error

Figure 3: ROC curve with neural network results.

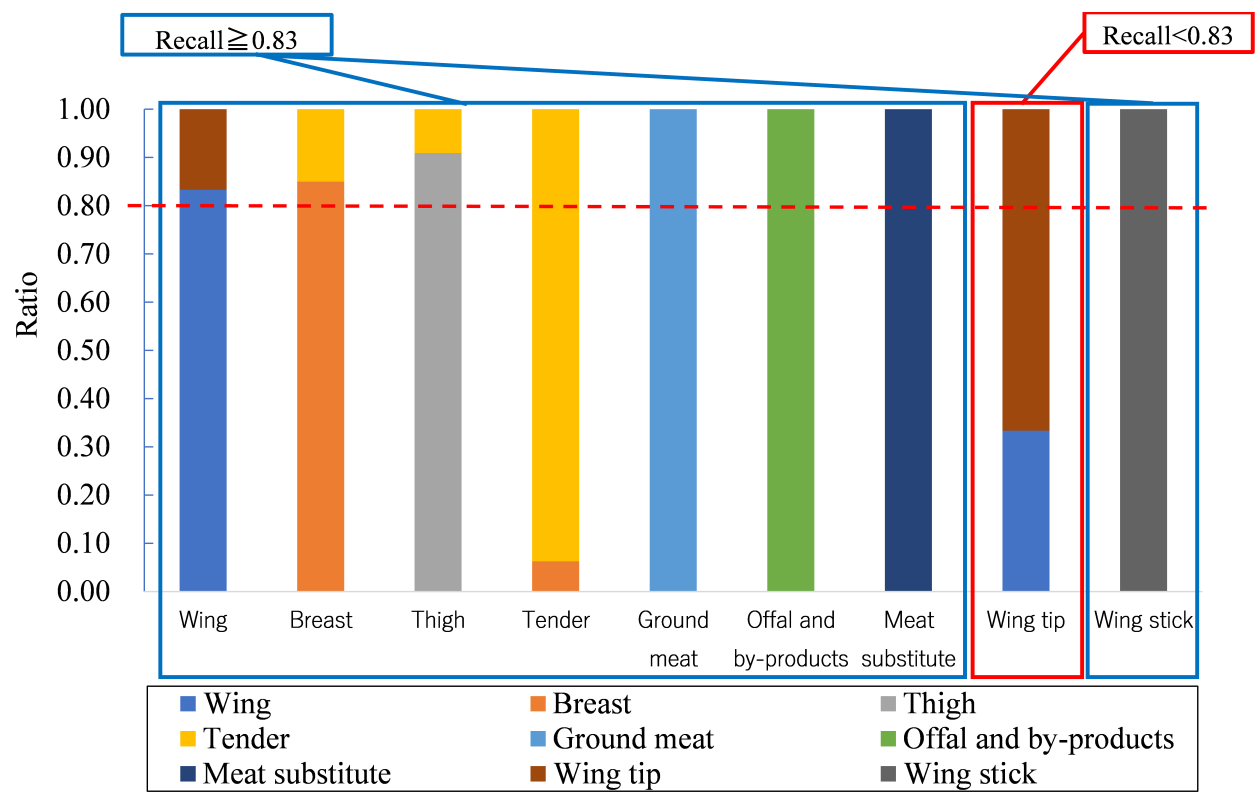

Figure 4: Results of neural network analysis using inorganic components in poultry products.

also inadequate for a model to determine unknown samples. In contrast, when considering measurement errors, the number of samples increases to 114 , which improves the positive discrimination rate and enables the creation of a model that can accurately determine the parts of poultry products.

Figure 3 shows the ROC curves for each measurement result: (a) without error and (b) with error. The ROC curve is created for each part, and the closer the area inside the curve is to 1 , the fewer false positives there are, indicating that the detector is suitable.

The results of the error-free data in Fig. 3(a) show that even the curve for "Thigh" with the largest area has $A=$ 0.925 , and the narrowest is $A=0.297$ for the "Wing stick".
The average was $A=0.736$, indicating that there were many misjudgments by the classification and matching model.

The results of the data with errors in Fig. 3(b) show that "Wing", "Offal and by-products", "Meat substitute", and "Wing tip" had $A=1.000$, indicating that they could be accurately classified and matched. Even the lowest value for "Tender" was $A=0.844$, which is higher than the average of the error-free data, $A=0.736$. The average for the model with errors is $A=0.976$, indicating that the model is accurate as a classification and matching model.

Figure 4 shows the predicted recall ratios for each part of the meat. The results show that the predic- 
tion ratios of "chicken wing", "breast", "thigh", "tender", "minced meat", "offal and by-product", "substitute meat" and "chicken wing" were higher than Recall=0.83. However, only "chicken wings" had a slightly lower predicted reproduction ratio of Recall $=0.67$.

In addition to the samples used in the aforementioned neural network, 76 types of samples were created by varying the inorganic component values of each part within a range of $\pm 10 \%$ and incorporated into a neural network.

The results show that the neural network correctly predicted 72 out of 76 dummy data points for available parts. The correct discrimination rate was $94.7 \%(\fallingdotseq 72 / 76)$, which indicates that we can obtain correct detection results by increasing the number of samples for the neural network.

\section{Conclusion}

In this report, we presented the possibility of classifying and matching poultry products using inorganic components that can quantify. In the future, we will examine the hidden layer and training iterations to improve the classification matching accuracy for each category. We will determine to which category products with an unknown classification belong.

\section{Acknowledgment}

This research was partially supported by the Ministry of Education, Science, Sports and Culture, Grant-in-Aid for Scientific Research (C), 2018-2021(18K02210, Yoichiro Kojima).

\section{References}

[1] Ministry of Education, Culture, Sports, Science and Technology, Food ingredient database, https://fooddb.mext. go.jp/, access date: 2020.10.12 (In Japanese)

[2] Japan Food Research Laboratories, "Component Analysis Point for Nutrition Labeling", Central Regulation, Vol., pp.116-140, 2007 (In Japanese).

[3] O. Akihiro, "Determinants of the Deliciousness of Meat", Japanese journal Nutrition Journal, Vol.60, No.3, pp.119129, 2002 (In Japanese).

[4] Y. Kimura, Y. Kojima, T. Mikami and K. Utsuno, "Classification of beef using food composition data and discriminate analysis", Japanese journal of taste and smell research, Vol.18, No.3, pp.443-446, 2011 (In Japanese).

[5] M. Goda and Y. Kojima, "Study on Classification Possibility of Pork Products by Chemometrics", Japanese Journal of the Institute of Industrial Applications Engineers, Vol.6, No.1, pp.33-43, 2018 (In Japanese). DOI: 10.12792/jjiiae.6.1.33

[6] M. Goda and Y. Kojima, "Possible classification of pork products by ICP-MS", Journal of the Institute of Industrial Applications Engineers, Vol.8, No.2, pp.46-49, 2020. DOI: 10.12792/JIIAE.8.46

[7] M. Goda, Y. Kojima and S. Iwanami, "Examination of beef products classification using linear regression analysis", The Japanese Journal of the Institute of Industrial Applications
Engineers, Vol.8, No.1, pp.53-57, 2020 (In Japanese). DOI: 10.12792/jjiiae.8.1.53

[8] W. S. Mcculloch and W. Pitts, "A logical calculus of the ideas immanent in neurons activity", The bulletin of mathematical biophysics, Vol.5, No.4, pp.115-133, 1943. DOI: 10.1007/BF02478259

[9] L. Zhang and B. Zhang, "A geometrical representation of McCulloch-Pitts neural model and its applications", IEEE transactions on neural networks, Vol.10, No.4, pp.925-929, 2002. DOI: $10.1109 / 72.774263$

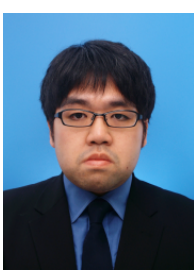

Motoki Goda (Member) In March 2015, he completed the master's program at the Graduate School of Environmental Science, Hokkaido University, and received a master's degree in environmental science. In the same year, he joined North Japan Port Consultants Co., Ltd. In April 2017, he was appointed as a researcher at the Hokkaido University of Science. In March 2021, he received a $\mathrm{Ph}$. D. He is engaged in research for port planning, behavior factor analysis, food quality control using AI/IoT and chemical component value information, and the identification of deliciousness factors.

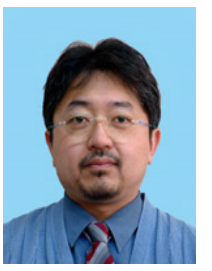

Shunsuke Iwanami (Non-member) In 1996, he completed the Doctoral Program in Agrochemistry, Faculty of Agriculture, Hokkaido University. Currently, he is a professor in the Department of Engineering for Innovation, at the National Institute of Technology, Tomakomai College. He is consistently engaged in research on the processing of ingredients produced in Hokkaido using biological functions, fermentation brewing, such as fruit vinegar, fruit wine, fish soy sauce, cheese, etc.

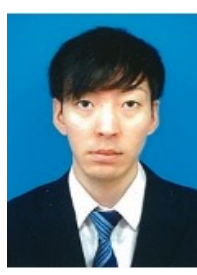

Nomura Akihiro (Non-member) In March 2015, Graduated from the Faculty of Engineering, Tokushima University. In the same year, he joined North Japan Port Consultants Co., Ltd. $\mathrm{He}$ is engaged in research on the program and mathematical Model that calculates the development of secondary circulation in meandering channel flow and characteristics of the explosive cyclone passing around Hokkaido using d4pdf, long-term evaluation of wave preproduction around Hokkaido by statistically.

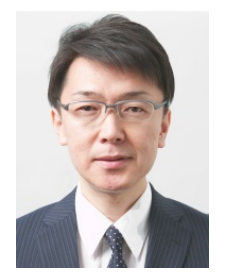

Yohichiro Kojima (Member) He received his $\mathrm{BE}, \mathrm{ME}$ and Dr. Eng. degrees, all in applied electronics engineering, from the Hokkaido Institute of Technology in 1990, 1992 and 1995, respectively. He is now a Professor at the Department of Electrical and Electronic Engineering, Faculty of Engineering of Hokkaido University of Science. His main research interests are focused on the development of taste sensing. Dr. Kojima is a member of the Institute of Industrial Applications Engineers. 